\title{
¿“Corruptos» o «eficientes»? La gestión de combustible de la Policía Nacional en Lima
}

\author{
José Manuel Villaorduña*
}

HANS BÜRKLI**

* Magister en Sociología por la Pontificia Universidad Católica del Perú. Actualmente, estudiante de Maestría en Criminología en la Universidad Nacional del Litoral (Argentina). Docente en la Universidad Nacional Pedro Ruiz Gallo y en la PUCP. Correo electrónico: jvillaorduna@gmail.com

** Licenciado en Sociología por la Pontificia Universidad Católica del Perú. Actualmente, estudiante de Maestría en Política y Gestión de la Innovación en Ciencia y Tecnología de la PUCP. Correo electrónico: hburkli@pucp.edu.pe

Fecha de recepción: 05/09/2018. Fecha de aceptación: 16/06/2019 


\title{
¿“Corruptos» o «eficientes»? La gestión de combustible de la policía nacional en Lima
}

\section{RESUMEN}

El presente trabajo analiza los casos de uso ilícito del combustible por miembros de la Policía Nacional del Perú (PNP) en Lima, describiendo dicho uso como un fenómeno generalizado y normalizado al interior de la organización. Esta aproximación al problema explicaría por qué, a pesar de la implementación de sistemas de monitoreo y fiscalización del uso de combustible, los casos de sustracción y uso indebido no cesan, en tanto dichas prácticas se originan y sostienen en deficiencias estructurales y características culturales propias de la organización. La investigación procura dar cuenta de cuáles son las características y mecanismos que permiten la generalización de la actividad corrupta, destacando aquellas que legitiman dichas prácticas en las narrativas de sus miembros. Se explica la valoración positiva del uso ilícito del combustible en su aparente funcionalidad para el cumplimiento de la misión organizacional. De esta manera, se problematizan las definiciones tradicionales sobre la corrupción en el sector público y sus consecuencias. Para lograr los objetivos planteados se ha utilizado tanto información proporcionada por la Dirección de Logística de la PNP (DIRLOG) como testimonios recopilados en entrevistas con miembros de la organización.

Palabras clave: corrupción, Policía Nacional del Perú, normalización, cultura organizacional.

\section{"Corrupt» or "efficient»? The normalization of the improper use of fuel in the police organization of Lima}

\begin{abstract}
This paper examines cases of illicit fuel use on the part of members of the Peruvian National Police (PNP) in Lima, by regarding the phenomenon as part of the generalized and normalized corruption within the institution. This approach accounts for the failure of the implemented oversight systems to regulate fuel usage and the persistence of irregular practices, such as fuel extraction and unauthorized vehicle use given that these practices have their origin in and in turn uphold structural deficiencies and cultural characteristics of the institution. This research provides an account of the characteristics and mechanisms that allow the generalization of corruption, highlighting those activities that legitimize said practices among members' narratives. As a result, a positive appraise of illicit fuel use has been constructed in relation to an apparent fulfillment of the institutional mission. Following this approach, traditional definitions of corruption in the public sector and their consequences are problematized here. Information examined in this paper comes from the Direction of Logistics of the PNP (DIRLOG) and from testimonies collected during interviews with members of the organization.
\end{abstract}

Keywords: corruption, National Police of Peru, normalization, organizational culture. 


\section{INTRODUCCIÓN}

¿Es posible seguir confiando en una organización encargada de vigilar el cumplimiento de la ley cuando nos enteramos de que sus miembros trasgreden de manera consistente las normas que deberían regir sus actos? Asimismo, ¿es posible que dicha organización goce de legitimidad a ojos de la sociedad, e incluso de sus propios miembros? Ambas preguntas surgen dentro de un contexto en el que la ciudadanía considera a la corrupción como uno de los principales problemas sociales del país junto con la delincuencia; a la par de ello, se percibe a la Policía Nacional del Perú (PNP en adelante) como la tercera organización más corrupta del Estado ${ }^{1}$ (Proética, 2017).

Este trabajo se aproxima al fenómeno de la corrupción policial, centrándose en el caso particular de corrupción por el uso indebido del combustible asignado a los vehículos policiales. A raíz de las denuncias realizadas por algunos medios de comunicación en los últimos años, se ha expuesto un uso arbitrario recurrente de la dotación de combustible para fines diferentes de los establecidos formalmente por la organización, que van desde la reparación de vehículos inoperativos hasta la apropiación personal del producto de la venta del combustible (La República, 2017). Y estos casos continúan pese a la implementación de mecanismos de supervisión y control, como el Sistema Multiflota.

De esta manera, los objetivos del presente trabajo serán determinar en qué medida esta forma de corrupción está generalizada dentro de la organización policial de Lima e identificar cuáles son los mecanismos que permiten que tales actividades corruptas persistan en la PNP sin deslegitimar la autoridad ni afectar la imagen que tienen los policías sobre sí mismos. Para alcanzar tales metas, se ha construido un indicador de confiabilidad en los reportes, que evalúa la información suministrada por los efectivos policiales durante el proceso de abastecimiento de combustible en Lima, y se ha recopilado una serie de entrevistas a efectivos policiales que exponen cómo es que se normaliza este tipo de corrupción al interior de la organización policial y cuáles son los mecanismos de control social al interior de la PNP que garantizan la continuidad de la práctica.

La corrupción es uno de los problemas que más afecta a la sociedad no solo por provocar grandes pérdidas económicas y sociales inmediatas (Dacoba, 2009), sino también por generar una crisis de legitimidad en sus instituciones. En el caso del uso ilícito del combustible en la PNP y sus efectos en las finanzas de la organización, como en la imagen que de esta última se forma la población, la mayoría

\footnotetext{
Según la encuesta Proética 2017 sobre las percepciones que se tienen en el Perú sobre la corrupción, la Policía Nacional es vista como la tercera institución más corrupta del país, solo superada por el Poder Judicial y el Congreso de la República.
} 
de los esfuerzos por comprender y combatir este fenómeno se han realizado desde enfoques moralistas o legales que conciben los actos de corrupción policial como casos de «manzanas podridas» ${ }^{2}$ que deben ser detectadas y erradicadas de la organización por corromper su normal funcionamiento (Trevińo y Youngblood, 1990). Estas aproximaciones no consideran que algunas actividades corruptas puedan estar normalizadas o institucionalizadas dentro de la organización por el rol que desempeñan al interior de ella. En ese sentido, este trabajo contribuye a comprender las estructuras y dinámicas concretas del funcionamiento de la organización policial en el departamento de Lima, así como el papel que cumple la corrupción en pos de dicho funcionamiento.

\section{LOS TIPOS DE CORRUPCIÓN POLICIAL}

Es difícil encontrar una definición de corrupción o corrupción policial precisa debido a las diferentes configuraciones que tales prácticas toman según el contexto sociohistórico en el que ocurren. Por ello, se entenderá a las prácticas corruptas como fenómenos particulares que poseen sus propias lógicas, mecanismos, contextos y configuraciones organizacionales (Huber, 2008; Boehm y Lambsdorff, 2009). Sin embargo, se pueden resaltar elementos en común en las definiciones esbozadas por la mayoría de trabajos e investigaciones académicas: el abuso del poder público, el beneficio privado, así como el deterioro de la legitimidad de la autoridad y del interés común (Nye, 1967; Tanzi, 1998; Soto, 2003).

Es posible, por lo tanto, elaborar una definición de la corrupción en el sector público como un fenómeno que comprende el uso indebido del poder por representantes políticos o funcionarios de la burocracia estatal, con el objetivo de obtener diversas formas de retribuciones, sean económicas o no, para uso particular, afectando el interés general en beneficio de un interés particular y socavando la legitimidad de la autoridad. No obstante, aunque esta definición podría encontrar consenso entre algunos autores dedicados al tema (Nye, 1967; Quiroz, 2013; Soto, 2003; Tanzi, 1998), otro sector podría discutir la oposición asumida entre corrupción y bien común. Autores como Ludwig Huber (2008) o Colin Leys (1965) señalan que la corrupción no siempre ha sido un mal a priori en las sociedades, pues en muchas ocasiones ha cumplido roles importantes dentro de ellas, como corregir fallas del mercado o de las burocracias estatales. Debido a estas consideraciones, resulta oportuno presentar una tipología de la corrupción policial según los medios, fines, motivaciones, formas en que se manifiestan y sus relaciones con

2 Casos poco frecuentes donde una minoría de oficiales incurre en actos de corrupción debido a su baja calidad moral. 
el bien común. Tal tipología tiene como base los ocho tipos de corrupción policial presentadas por Roebuck y Barker (1974), y se incluye además el concepto de corrupción blanca, desarrollado por Kleining (2002).

Tabla 1. Tipología de actos de corrupción policial

\begin{tabular}{ll}
\hline Tipo & Descripción \\
\hline Sobornos internos & $\begin{array}{l}\text { Compra, intercambio o venta de privilegios entre los policías. } \\
\text { Se crea un mercado en que los actores son solo policías. }\end{array}$ \\
\hline $\begin{array}{l}\text { Actividades criminales } \\
\text { directas }\end{array}$ & $\begin{array}{l}\text { En este tipo de corrupción policial no hay la figura de un } \\
\text { corruptor. Refiere a la realización de crímenes por la policía } \\
\text { contra personas u organizaciones para beneficio personal. }\end{array}$ \\
\hline Arreglos & Debilitamiento adrede de casos de investigación criminal. \\
\hline $\begin{array}{l}\text { Protección de actividades } \\
\text { ilegales }\end{array}$ & $\begin{array}{l}\text { Protección policial de personas involucradas en actividades } \\
\text { ilegales, de manera que se les permita seguir operando. }\end{array}$ \\
\hline $\begin{array}{l}\text { Pedido o aceptación de } \\
\text { sobornos }\end{array}$ & $\begin{array}{l}\text { Chantajes que surgen de manera oportunista para no actuar } \\
\text { frente a una falta o un acto criminal. Este tipo de actos } \\
\text { incluyen el pedir o aceptar dinero a cambio de no imponer } \\
\text { una multa de tránsito, o para no proceder al arresto de un } \\
\text { criminal }\end{array}$ \\
\hline $\begin{array}{l}\text { Robrupción por causa } \\
\text { noble o corrupción blanca } \\
\text { (Kleining, 2002) }\end{array}$ & $\begin{array}{l}\text { Actuar contra los deberes de la organización para asegurar } \\
\text { un bien superior, como la seguridad pública. No buscan el } \\
\text { beneficio privado del efectivo policial, sino más bien están } \\
\text { orientadas hacia el bien público. }\end{array}$ \\
\hline $\begin{array}{l}\text { fickback }) \\
\text { no autorizada de un botín abandonado o evidencia } \\
\text { confiscada. }\end{array}$ \\
\hline $\begin{array}{l}\text { Cuando el efectivo policial recibe bienes, servicios o dinero } \\
\text { por derivar servicios a terceros con afán de hacer negocios con } \\
\text { las personas intervenidas durante el patrullaje. }\end{array}$ \\
\hline $\begin{array}{l}\text { Recepción de una ganancia no autorizada por el policía solo } \\
\text { por su posición, sin violar ninguna ley per se. }\end{array}$ \\
\hline
\end{tabular}

Fuente: Roebuck y Barker (1974). Elaboración propia.

Como ya se mencionó, algunos autores sostienen que no todo acto de corrupción es condenable ni perjudicial a priori y, en el caso específico del tipo de corrupción blanca, incluso se le puede entender como una respuesta comprensible a las complejidades del mundo y a la dificultad que implica desarrollar reglas que se acomoden a cada exigencia (Kleining, 2002; Huber, 2008).

Las organizaciones, incluida la Policía Nacional, no solo operan en función de las instituciones formales de la organización, así como de las normas codificadas y 
comunicadas abiertamente como oficiales, sino también de acuerdo con instituciones informales, reglas socialmente compartidas que son creadas, comunicadas y aplicadas fuera de los canales de sanción establecidos formalmente (Acha, 2004; Helmke y Levitsky, 2003). El conjunto de estas reglas o instituciones informales que no provienen de reglamentos oficiales, pero que sí están presentes en las dinámicas organizacionales reales, es denominado "cultura organizacional» (Toca y Carrillo, 2009). La cultura organizacional surge de las prácticas al interior de la organización, pero también del marco social, político y económico dentro del cual está inscrita. En ese sentido, la cultura policial está influenciada por la labor policial misma, pero también por el contexto donde se sitúa (Chan, 1996), y la respuesta de la organización a dicho entorno (Acha, 2004). Así, se entiende que, pese a que las funciones formales de las organizaciones policiales de diversos países son similares, sus características reales difieren entre sí.

Es importante mencionar algunas instituciones informales que son comunes en diferentes experiencias policiales, pues entreven los elementos culturales que permiten que se sostenga la actividad corrupta que analizaremos. En primer lugar, el "código de silencio» consiste en no informar o reportar conductas indebidas de otros efectivos policiales ${ }^{3}$ (Tudela, 2010). En segundo lugar, el abuso de la discrecionalidad, que, si bien es una característica necesaria para la aplicación de las normas por diversos funcionarios, es más utilizada y requerida por los policías, pues sin ella no lograrían cumplir sus funciones (Reiner, 2010). Por último, la verticalidad característica de toda organización fuertemente jerarquizada, que implica la obediencia de los miembros hacia sus superiores. Cabe resaltar que, aunque la subordinación esté enmarcada dentro de reglas formales, muchas veces se desbordan los límites establecidos por ellas y se procede a obedecer «sin dudas ni murmuraciones» (Acha, 2004).

Las prácticas corruptas se generalizan al interior de una organización mediante un proceso de normalización, a través del cual ideas y acciones que pueden ser vistas como ilícitas o inmorales pasan a ser vistas como normales y legítimas al interior de un grupo (May, 2009). La aproximación al proceso de normalización de la corrupción en el caso de la dotación de combustible en la PNP se sustenta en el trabajo de Ashforth y Anand (2003). Dichos autores plantean que detrás de todo proceso de normalización de actividades corruptas se dan tres procesos que se retroalimentan y complementan de forma bidireccional al interior de la organización: institucionalización, racionalización y socialización. La institucionalización se refiere al momento

\footnotetext{
3 Algunos autores han seńalado que esto se debe a la solidaridad interna de grupo y también porque ofrece seguridad a sus miembros frente a situaciones de peligro propias del trabajo policial (Chan, 1996).
} 
en el cual un acto corrupto se incrusta en las estructuras y procesos de la organización con lo cual se vuelve rutinario y pasa a ser un elemento propio del grupo. La racionalización está ligada al momento en el cual se desarrollan estructuras ideológicas para justificar e incluso para valorizar la actividad corrupta. Por último, la socialización es el proceso mediante el cual nuevos participantes son inducidos a ver la corrupción como permisible y hasta deseable.

\section{Mapa 1}

Procesos para la normalización de la corrupción

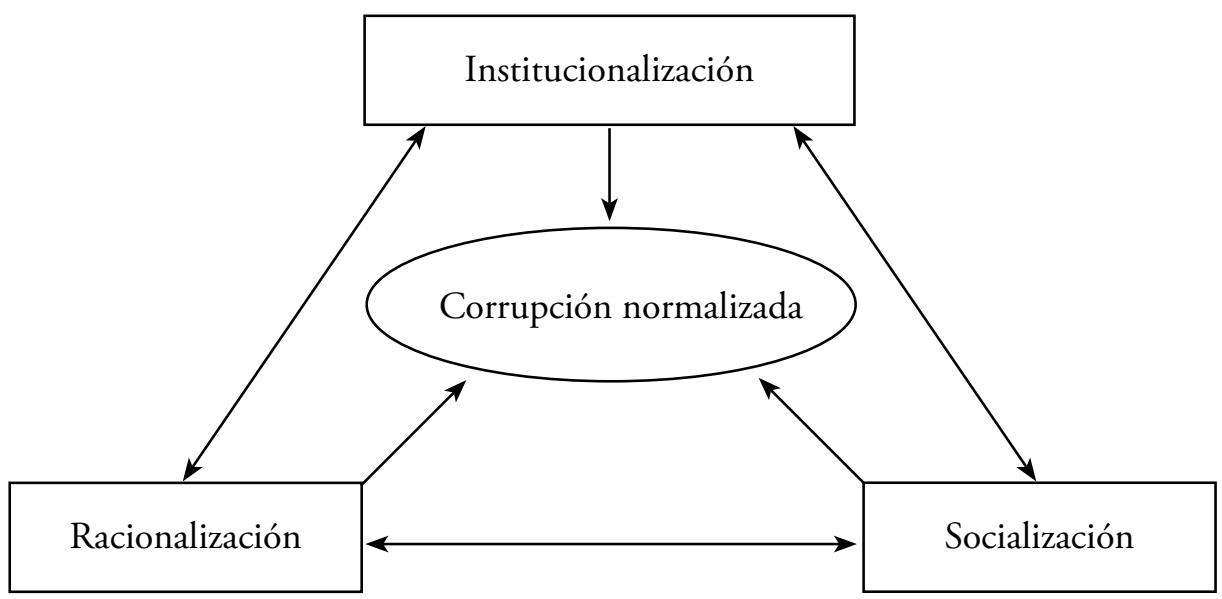

Fuente: Ashforth y Anand (2003).

\section{Metodología}

Este trabajo se centra en constatar la normalización en el uso ilícito del combustible en la PNP de Lima Metropolitana entre 2014 y 2015. Dicho uso se pone en evidencia con la implementación del Sistema Multiflota, que empezó a operar en Lima Metropolitana en 2014 como un sistema de control de abastecimiento para la administración de conductores y vehículos de la flota de la PNP. Según este sistema, cada oficial responsable de un vehículo policial debe identificarse ante el despachador del grifo, quien pasa la Tarjeta Multiflota en un dispositivo de cobranza $\left(\mathrm{POS}^{4}\right)$ para verificar que el oficial esté asociado a la unidad correspondiente; luego de ello, digita en el sistema el kilometraje señalado por el odómetro ${ }^{5}$ del vehículo,

\footnotetext{
Point of Sale o máquina de punto de venta.

Dispositivo que marca la distancia recorrida por un vehículo, se usa generalmente para calcular tiempos de viaje, distancia recorrida o consumo de combustible.
} 
abastece al vehículo de combustible y le entrega una copia del recibo al efectivo policial, indicando la cantidad de galones surtidos.

El Sistema Multiflota, que permite supervisar las cifras de consumo y de usuarios, se diseñó como una medida para combatir la corrupción policial, se implementó primero en Lima y luego se expandió al resto del país ${ }^{6}$. De esta manera, se busca reducir este tipo de corrupción por medio de un sistema de monitoreo que debería elevar la posibilidad de detectar a los infractores. Este trabajo se enfocará en la PNP de Lima Metropolitana, el primer lugar en el que se implementó el Sistema Multiflota, para evaluar cómo la introducción de una nueva tecnología ha permitido evidenciar una práctica oculta. Además, gracias a su uso se dispone de los registros digitales de los procesos de abastecimiento en Lima Metropolitana, como insumo para analizar el nivel de generalización de la apropiación del combustible en la organización policial.

El presente trabajo analizará la información registrada en el Sistema Multiflota por los encargados de los vehículos de Lima Metropolitana entre 2014 y 2015, para comprobar la veracidad de la información. Teniendo en cuenta la base de rangos de rendimientos establecidos por la directiva 04-16-2011-DGPNP/DIRLOG-PNP/ DIVCOM se ha logrado construir un indicador que muestra cuán confiables son los datos registrados en el Sistema Multiflota. La Directiva señalada indica el rango de kilómetros que un vehículo policial debería recorrer por cada galón de combustible según el tamańo de su cilindrada ${ }^{7}$. De esta manera, la información registrada en el sistema — galones de combustible abastecidos y rendimiento del vehículodebería ser coherente con los rangos de rendimiento esperado para las características del vehículo en cuestión.

De suceder lo contrario, en la medida en que el rendimiento registrado se aleje significativamente del rendimiento esperado para el vehículo, se podría sospechar de la veracidad de la información ingresada al sistema y, por lo tanto, se puede presumir que se está frente a un acto de simulación del proceso. Establecemos que un reporte de rendimiento que se aleje entre $10 \%$ y $20 \%$ del rendimiento esperado será calificado como «sospechoso», mientras que uno que se aleje más allá del $20 \%$ sería «muy sospechoso». El indicador mostrará cuán común es la falsificación de información registrada en el Sistema Multiflota y, con ello, dará una idea de la generalidad del uso ilícito del combustible en la PNP de Lima Metropolitana en 2014 y 2015, es decir, si corresponde a un grupo minoritario de policías (las «manzanas podridas») o si es una práctica más bien generalizada en la PNP de Lima.

\footnotetext{
6 Con excepción del VRAEM.

7 La cilindrada, medida en centímetros cúbicos, es un buen indicador de la capacidad de trabajo que puede tener un motor. A mayor cilindrada menores serán los kilómetros recorridos por cada galón de combustible. Con esa información, la PNP construye una tabla de rendimientos esperados por tipo de vehículo.
} 
Una vez que se determine la extensión del fenómeno, se analizarán las entrevistas realizadas a agentes policiales para explicar los mecanismos al interior de la organización que hacen posible que dicha práctica exista y persista a través del tiempo. Debido al "pacto de silencio" y a la dificultad que implica conseguir información sobre delitos cometidos por los mismos entrevistados o sus compañeros, las entrevistas solo se realizaron luego de desarrollar relaciones de confianza con algunos miembros de la policía, dejando de lado cualquier selección por aleatoriedad ${ }^{8}$.

Los resultados y conclusiones de la presente investigación solo se circunscriben a la organización policial de Lima Metropolitana. Es posible que al interior del país existan otros mecanismos e instituciones informales sobre las cuales el uso ilegal del combustible se sostenga. Investigaciones futuras sobre la data en el país que ya recoge el Sistema Multiflota se podrán encargar de explorar las normas informales y transgresiones normalizadas que ocurran al interior del país.

\section{LA VENTA ILEgal DE COMbuSTible EN LA PNP}

Para cumplir sus funciones de garantizar, mantener y restablecer el orden interno', la PNP necesita una serie de elementos logísticos, uno de los cuales lo componen los vehículos patrulleros con los que cuenta la institución. La puesta en operación de todos esos vehículos constituye un sistema en sí mismo, dentro del cual opera otro subsistema, que es el sistema de abastecimiento de combustible. Para cumplir con la tarea de patrullaje, los vehículos requieren una dotación permanente de gasolina, por lo que, sobre la base de tal necesidad, se ha estructurado una serie de procesos para el abastecimiento que cuentan con su propia normativa y prácticas. A continuación, se describirá dicho sistema tanto en el campo de lo formal, o legal, como en el de las prácticas concretas.

\section{El sistema de abastecimiento de combustible y mantenimiento de la flota vehicular de la PNP}

La administración de combustible en la PNP se rige por la Directiva N 04 de la Dirección de Logística de la Policía Nacional (DIRLOG-PNP), la que establece un ciclo de abastecimiento que exige el cumplimiento de las siguientes etapas: requerimiento, adquisición y dotación. El presente trabajo se enfoca en la etapa de dotación ${ }^{10}$

\footnotetext{
8 Cabe resaltar que, para salvaguardar el bienestar de los entrevistados, todos los testimonios serán presentados anónimamente.

9 Artículo 166 de la Constitución Política del Perú.

10 No se ha considerado la etapa de adquisición de combustible porque es un proceso que tiene sus propias normas y se realiza de manera centralizada en la cúpula administrativa de la PNP. Tampoco se
} 
del sistema de abastecimiento de combustible, que es la etapa en la que los vehículos policiales deben ser conducidos a las estaciones de servicio para recibir la dotación de gasolina que tienen asignada, además del llenado de los sistemas de registro.

La norma describe detalladamente los pasos del ciclo de abastecimiento, y exige, para presuntamente garantizar el control y seguimiento del proceso, que los miembros de la policía encargados registren todas las acciones relativas a este. Entre los documentos de registro figuran las tarjetas de abastecimiento (TAC), los cuadernos de abastecimiento, los cuadernos de recorrido, etcétera. En Lima, las tarjetas multiflota reemplazaron a las tarjetas de abastecimiento, al implementarse un sistema que guarda digitalmente los datos del combustible abastecido, tales como lugar, fecha, hora, conductor responsable del abastecimiento y cantidad abastecida (Ministerio del Interior, 2014). Cabe resaltar que los datos deben ser introducidos manualmente al sistema digital por los encargados de los vehículos.

Así, en líneas generales, el sistema de abastecimiento formal es bastante minucioso, y exige el cumplimiento de varios registros para cruzar información y verificar que la dotación se haya realizado de acuerdo con las normas establecidas, ya que de suceder lo contrario, la actividad es considerada una infracción administrativa (código MG11; código MG-60) ${ }^{11}$.

Dadas sus funciones relativas a garantizar el orden interno, la vigilancia en las calles por medio del patrullaje es una de las principales actividades que debe llevar a cabo la PNP. Para asegurar el patrullaje, los vehículos policiales requieren de serie de insumos, uno de los cuales - pero no el único- es el combustible. Sin embargo, al examinar los datos ofrecidos por la Dirección de Logística de la PNP se evidencia la falta de correspondencia entre la adquisición de combustible y la de otros elementos necesarios para la operatividad de la flota vehicular policial, como llantas nuevas, lubricantes, aceite, etcétera. En 2014, la PNP asignó alrededor de 21331835 galones de combustible para sus vehículos en el país. De otro lado, la directiva 56-95-DGPNP-DIRLOG-DIMAN establece que a cada vehículo le corresponde un juego de llantas nuevo cada año o cada 80000 a 100000 kilómetros recorridos. De esta manera, según la proyección de kilómetros a recorrer por cada galón de

\footnotetext{
va a estudiar la etapa de requerimiento, ya que los pedidos de combustible varían poco con los años y responden de la misma manera a decisiones centralizadas.

11 «No respetar los procedimientos establecidos o alterar, distorsionar, encubrir o suprimir, sin motivo justificado, los documentos que sustentan el procedimiento regular de un proceso interno o pago a personal de la Policía Nacional del Perú o a un tercero» (código MG11). «Distorsionar, adulterar o suscribir información falsa en informe, certificado, peritaje u otro documento en beneficio propio o de terceros" (código MG-60). Disponible en el anexo III tabla de Infracciones y Sanciones Muy Graves, del decreto legislativo 1150 que regula el régimen disciplinario de la PNP, reglamentado por decreto supremo 013-2016-IN.
} 
combustible $(71,88 \mathrm{~km})^{12}$, se estima que al finalizar el año 2014 correspondía comprar 30181 llantas para toda la flota vehicular. Sin embargo, según a la información provista por la DIRLOG-PNP, ese año solo se distribuyeron 486 llantas en el país, lo que significa un déficit estimado de 30181 llantas en 2014, tal como muestra la tabla 2.

Tabla 2. Requerimientos y distribución de insumos para los vehículos de la PNP 2014

\begin{tabular}{|c|c|c|c|}
\hline $\begin{array}{l}\text { Dotación anual de } \\
\text { galones de combustible } \\
\text { (Lima y provincias) }\end{array}$ & $\begin{array}{c}\text { Kilómetros recorridos } \\
\text { estimados } \\
(71,88 \text { km/galón })\end{array}$ & $\begin{array}{l}\text { Llantas } \\
\text { requeridas }\end{array}$ & $\begin{array}{l}\text { Llantas } \\
\text { compradas } \\
\text { (unidades) }\end{array}$ \\
\hline 21331835 & 1533348419 & 30667 & 486 \\
\hline
\end{tabular}

Fuente: Proyecciones sobre la base de datos de DIRLOG PNP y directiva N 56-95-DGPNP-DIRLOG. Elaboración propia.

Los datos muestran dos realidades diferentes en torno a la distribución de insumos necesarios para la operatividad de la flota vehicular policial. Por un lado, no se adquieren llantas ${ }^{13}$ en cantidades suficientes a las requeridas por la organización policial; en contraste, se evidencia una dotación garantizada y constante de combustible. Sí se reconoce que, pese a que únicamente se atendieron las necesidades de combustible y no otros requerimientos para la operatividad de los vehículos, como llantas y lubricantes, la flota vehicular se ha mantenido operativa, se vuelve interesante indagar sobre las prácticas concretas que se han construido alrededor de este insumo, para explicar cómo es que los vehículos policiales pueden funcionar pese a esta notable inconsistencia.

\section{Prácticas concretas de dotación de combustible en la PNP}

Según lo señalado por los policías entrevistados, varios miembros de la policía sustraen y venden el combustible otorgado para darle un uso diferente del establecido por las normas de la organización. Ello se da por medio de dos modalidades: el «vampireo» y la reventa de los saldos de combustible en el grifo concesionario.

\footnotetext{
12 Utilizando el cuadro de rendimientos de combustible según tipo de vehículo y tamaño de cilindrada, así como los datos de las cilindradas de cada uno de los vehículos de la flota de la PNP, se obtuvo un rendimiento promedio de 71,88 kilómetros por galón. Este alto rendimiento se explica en tanto el $57 \%$ de los vehículos policiales tenía una cilindrada inferior a los 1650 cc y el $43 \%$ tenía una cilindrada menor de $300 \mathrm{cc}$.

13 La situación es similar con otros insumos como galones de aceite para motor y filtros de aceite, de los cuales se requerían 103828 galones dado el recorrido estimado, sin embargo, solo se compraron 20000 galones de aceite y 3200 filtros.
} 
En el primer caso, los policías proceden a succionar, con el uso de mangueras, el combustible de los vehículos después de que estos ya han sido abastecidos. Luego de ello, el combustible es acopiado para su posterior venta. En el segundo caso, solo se entrega a los vehículos policiales una porción del total del combustible adquirido para todo el mes por la organización policial. Posteriormente, al final de cada día, semana o mes, dependiendo de lo que se haya acordado entre la estación de servicio y el jefe de la unidad policial, el representante del grifo paga al efectivo policial por la venta a terceros del combustible que no fue entregado a los vehículos policiales.

Como ya se señaló, el Sistema Multiflota surgió como una innovación que buscaba combatir este tipo de actividades corruptas. Sin embargo, tal como señalan varios medios de comunicación, estas actividades han persistido bajo nuevas modalidades como la simulación de abastecimientos (El Comercio, 2015; La República, 2017). Para conocer cuán generalizado está este tipo de prácticas corruptas por el uso ilícito del combustible dentro de la PNP, se ha utilizado la información registrada en el Sistema Multiflota en los años 2014 y 2015.

La normatividad del Sistema Multiflota establece la obligatoriedad del registro del kilometraje recorrido al momento del abastecimiento. Gracias a dicho registro, el sistema calcula el rendimiento de cada vehículo; es decir, calcula cuántos kilómetros habría un vehículo con la cantidad de combustible otorgada, considerando las características propias del vehículo, específicamente el tamaño de su cilindrada ${ }^{14}$. Un indicador de que los vehículos no están presentes en el grifo al momento de registrarse el abastecimiento, y, por lo tanto, un indicador de que no se está abasteciendo el combustible, es cuando los datos de rendimiento son incoherentes con la realidad. Hemos recopilado algunos de estos casos en la tabla 3.

Tabla 3. Reporte de rendimientos del combustible irregulares en unidades policiales de Lima y Callao

\begin{tabular}{|c|c|c|c|c|c|}
\hline Hora & Marca & $\begin{array}{l}\text { Kilometraje } \\
\text { registrado }\end{array}$ & $\begin{array}{c}\text { Tipo de } \\
\text { combustible }\end{array}$ & $\begin{array}{c}\text { Galones } \\
\text { Abastecidos }\end{array}$ & $\begin{array}{c}\text { Rendimiento } \\
\mathrm{km} / \mathrm{Gl} . \mathrm{O} \mathrm{km} / \mathrm{m}^{3}\end{array}$ \\
\hline 07:20:04 & Hyundai & 0 & Gasolina 90 & 6 & 0 \\
\hline $21: 41: 27$ & Yamaha & 30878 & Gasolina 90 & 1,5 & 19468 \\
\hline $21: 14: 29$ & Toyota & 57674 & Gasolina 90 & 8 & $-64618,38$ \\
\hline $11: 44: 17$ & Toyota & 574735 & Gasolina 90 & 8 & 64632,63 \\
\hline $16: 54: 54$ & Nissan & 447122 & DB5 S50 & 8 & 55890,25 \\
\hline
\end{tabular}

Fuente: División de Combustible PNP. Elaboración: Inspectoría General del Sector Interior.

14 La cilindrada es una forma de representar el tamaño del motor. Es una medida que expresa el total del volumen de todos los cilindros del motor. Por ser una medida de volumen, la cilindrada se expresa en centímetros cúbicos o litros. 
Como se observa, rendimientos de 0 kilómetros por galón de combustible, rendimientos negativos y rendimientos exageradamente elevados dan cuenta del registro de un número al azar al momento del abastecimiento y, por lo tanto, son un indicador de que, al momento en que se registró el abastecimiento, el vehículo no estuvo presente en el grifo. Es decir, los datos mostrados en la tabla evidencian la simulación del cumplimiento de las normas con el fin de dar al combustible un uso diferente del establecido.

De esta manera, al contrastar los rangos de rendimientos esperados para cada tipo de vehículo, considerando el tamaño de su cilindrada, establecidos por la directiva 04-16-2011-DGPNP/DIRLOG-PNP/DIVCOM y los rendimientos registrados en el Sistema Multiflota, el indicador de confiabilidad construido arroja los siguientes resultados sobre el total de registros realizados en el Sistema Multiflota para el período 2014-2015 en Lima Metropolitana.

Tabla 4. Clasificación de rendimientos de combustible según tipo de vehículo e indicador de sospecha de reportes de rendimiento según rangos (2014-2015)

\begin{tabular}{|c|c|c|c|}
\hline $\begin{array}{l}\text { Reporte de rendimiento de } \\
\text { combustible }\end{array}$ & $\begin{array}{l}\text { Calificación de la } \\
\text { información }\end{array}$ & $\begin{array}{l}\text { Cantidad de } \\
\text { reportes }\end{array}$ & $\begin{array}{l}\text { Porcentaje } \\
\quad(\%)\end{array}$ \\
\hline Dentro del rango $+/-10 \%$ & Reportes normales & 441895 & 32,08 \\
\hline $\begin{array}{l}\text { Entre } 10 \% \text { y } 20 \% \text { mayor o menor } \\
\text { al rango }\end{array}$ & Reportes sospechosos & 128515 & 9,33 \\
\hline \multirow[t]{2}{*}{$\begin{array}{l}\text { Mayor o menor al rango en más } \\
\text { de } 20 \%\end{array}$} & $\begin{array}{c}\text { Reportes altamente } \\
\text { sospechosos }\end{array}$ & 806999 & 58,59 \\
\hline & Total & 1377409 & 100 \\
\hline
\end{tabular}

Fuente: Sistema Multiflota. Elaboración propia.

Como se puede observar, en una muestra de 5031 vehículos policiales, entre septiembre de 2014 y diciembre de 2015, se registraron 1377409 reportes de abastecimiento de combustible en el Sistema Multiflota. Aplicando la clasificación propuesta para el rendimiento registrado del combustible, se evidencia que la mayoría de abastecimientos son altamente sospechosos y se podría intuir que se trata de casos donde se da un uso irregular al combustible asignado. Solo el 32,08\% de los reportes cae dentro de los rangos esperables de rendimiento ${ }^{15}$ calculados por la Dirección de Logística PNP, de acuerdo con el tipo de vehículo y su cilindrada.

\footnotetext{
15 Sin embargo, el hecho de que un reporte arroje un rendimiento normal no es una garantía de que dicho abastecimiento fue efectivamente realizado: solo indica que los datos ingresados de kilometraje recorrido son consistentes con los rendimientos esperados.
} 
Así, el 58,59\% de los reportes responden al registro de datos falsos durante el abastecimiento de combustible.

Por lo expuesto, se puede afirmar que, pese a lo que se observa en varios reportajes periodísticos (América TV, 2014; El Comercio, 2011), la falsificación y simulación de la información requerida para el abastecimiento de los vehículos, y por lo tanto el uso irregular del combustible, no es un delito cometido esporádicamente y por unas cuantas «manzanas podridas» dentro de la organización, sino que es una actividad generalizada en la PNP.

\section{La normalización del uso indebido de combustible en la PNP}

$\mathrm{Al}$ estar garantizado el suministro del combustible y no el de otros insumos necesarios, la propia organización policial termina creando las condiciones para que sus integrantes se salten las normas, o cuando menos, les proporciona una justificación para tales acciones. De acuerdo con los testimonios recogidos, los policías venden el combustible, y con el dinero obtenido, subsanan las carencias de la unidad policial, ya que de otra manera no se podría garantizar el patrullaje. Así, la distribución deficiente de recursos para el mantenimiento de la flota vehicular es el punto de partida de la narrativa que justifica los actos de transgresión. Al respecto, son relevantes los siguientes testimonios:

Yo tengo el concepto de una mala administración [...] Por ejemplo, no sé cuánto tiempo tienen los patrulleros inteligentes circulando, será uno o dos años, y técnicamente al ańo ya deberían haber cambiado las llantas, ¿̨les habrán comprado a los vehículos de todas las unidades operativas llantas? Y qué implica esto: que obviamente, se va a tener que buscar la manera de comprar llantas, de repente de segunda, ni siquiera nuevas, con la gasolina. Es algo que se tiene que hacer. (Informante 10, 2015)

Entonces eso sería lo formal, pero ya hablando de lo informal, entonces ¿qué cosa es?: tener que utilizar el mismo combustible, tratar de racionalizar, entre comillas, para poder, con eso mismo, reparar tu vehículo patrullero, que es el problema. (Informante 1, 2014)

Los miembros de la PNP que incurren en estas prácticas no se ven a sí mismos de la manera en que la prensa y la opinión pública los señala. Sus propias narrativas los describen como funcionarios que, dadas las carencias de la organización, se ven obligados a vender el combustible para poder cumplir con sus funciones. Existe por lo tanto una disonancia entre la percepción que los policías tienen de sí mismos frente a la percepción que tiene la sociedad de ellos. Para explicar cómo esta diferencia de percepciones ha sido posible —es decir, de qué manera las actividades calificadas como corruptas por el uso ilegal del combustible han encontrado 
legitimidad al interior de la organización policial—, partimos de lo propuesto por Ashforth y Anand (2003) sobre los procesos complementarios que conducen a que actos ilícitos y supuestamente inmorales pasen a ser vistos como normales y cotidianos, a saber, los procesos descritos por dichos autores como institucionalización, racionalización y socialización.

Según la propuesta de Ashford y Anand (2003), la institucionalización se define como la sucesión de acciones que llevan a que un acto corrupto se incruste en las estructuras y procesos de la organización, con lo cual se vuelve rutinario y pasa a ser considerado un elemento propio del grupo (2003). En cambio, la racionalización referiría a los mecanismos por los cuales se justifica y valora la actividad corrupta. Sin embargo, en los casos que hemos estudiado encontramos que ambos procesos ocurren de manera simultánea, por lo que no encontramos relevante para el presente estudio la distinción analítica entre institucionalización y racionalización, de manera que serán considerados como un solo proceso.

El proceso de institucionalización y racionalización en la venta de combustible en la PNP se produce por la distribución dispar de recursos para el patrullaje, que lleva a que dicha práctica quede incorporada en los procesos organizacionales y permite la construcción de un discurso en el cual los actos corruptos cobran un sentido y una justificación para los actores involucrados. Al constatarse la carencia de recursos necesarios para el mantenimiento y funcionamiento de los vehículos policiales, queda claro al interior de la organización que tal situación debe ser remediada recurriendo a la venta del combustible. De esta manera, los mecanismos que permiten la extracción y venta del combustible se convierten en rutinarios y justificados a lo largo del grupo policial.

Te doy un ejemplo claro: un día el personal, son las siete de la mañana, sale a su servicio diario y resulta que no puede salir en razón de que el vehículo tiene un desperfecto mecánico. Se descubre en el momento que es la falla en la bomba de combustible: el vehículo está inoperativo y si sale va a salir a medias. Solución: hay que comprar otra bomba de combustible. ¿Cuánto vale? 150 soles, ¿Qué se hace en el momento? En el momento se trata de buscar una solución inmediata para que no se pierda un vehículo patrullero durante veinticuatro horas. ¿Cuál es la solución? Comprar la bomba. Entonces, si ese vehículo tiene seis galones diarios, ¿qué hago? Echo solo tres galones, $50 \%$ de recorrido de ese carro, que la tripulación lo sabe, y ahorro tres galones para comprar ese repuesto. Porque si lo hago a través de la formalidad, debo hacer un informe técnico, ir a la maestranza donde hay mecánicos de la policía, me digan cuál es la falla, la descubran, me hagan un informe técnico, ese informe lo elevo a mi comando y a la Dirección de Administración y Logística para la adquisición, que puede demorar diez días, veinte días, un mes, y así respondan que sí o que no se puede comprar, o que lo van a comprar después. Significaría que ese carro estaría parado todo ese tiempo. Entonces ¡cuál es mi capacidad de gestión? (Informante 2, 2014) 
El testimonio expone cómo la venta de combustible se sustenta además en un discurso sobre la necesidad de encontrar soluciones de manera inmediata, algo que una burocracia morosa y engorrosa, de acuerdo con lo expuesto por el informante, no permitiría. Del escenario descrito en los testimonios se puede inferir que el Comando Policial asume que las carencias al interior de la organización no solo pueden, sino que deben ser solucionadas, y de manera oportuna, por los respectivos jefes de unidad. Es decir, existiría una suerte de pacto no escrito en el que la plana mayor de la policía, a sabiendas de que existe una distribución dispar de los recursos destinados al mantenimiento de la flota vehicular, otorga responsabilidades y asigna una misión a los oficiales que están a cargo de las diferentes unidades de la PNP, confiando en que estos sabrán cómo agenciárselas para suplir dichas carencias:

Si ni siquiera dan para mantenimiento y me dicen «ustedes vean cómo manejan su administración». Te presionan la parte operativa, exigen resultados, uno trata de mantener su flota en funcionamiento, y como tú tienes que manejar tu zona como te lo exigen, te ves obligado a que tú mismo realices las reparaciones de manera particular, recurriendo a la gasolina. (Informante 9, 2015)

Asimismo, los miembros de la policía recurren a conceptos que vienen de la economía o de la gerencia de empresas para justificar la venta del combustible asignado para el patrullaje. Esto ocurre por lo general con oficiales de mayor rango, como comandantes, coroneles y generales, quienes utilizan términos como «eficiencia» o «racionalización de los recursos» para explicar que la venta del combustible para comprar repuestos no es solo una decisión perfectamente racional, sino que es el tipo de decisiones que se espera que tome un oficial superior:

Y eso sucede [la venta de combustible para comprar repuestos], eso es lo que hace una gestión y tiene la capacidad de gestión, pero hay que reconocerlo, no de la manera idónea, adecuada. (Informante 2, 2014)

De otro lado, Ashford y Anand también distinguen la socialización como el proceso mediante el cual se introduce a nuevos participantes a ver y valorar las actividades corruptas como permisibles y hasta deseables. En el caso del uso ilícito del combustible, una de las maneras en que se socializa tal transgresión es introduciendo a los nuevos oficiales en el sistema de administración de combustible desde sus primeros años: al otorgarles la administración de un vehículo que tendrá los mismos problemas de asignación dispar de recursos, deberán enfrentar el problema desde sus primeros años en el cuerpo policial. De esta manera, los oficiales entienden rápidamente desde la práctica cuáles son las carencias de la organización y la manera en que deben afrontarlas en lo sucesivo: 
Bueno, yo he trabajado en manejo de vehículos desde que era teniente, o sea quiere decir que un oficial desde sus primeros grados tiene por lo menos la administración de un vehículo, o sea, quiere decir que todo oficial va a pasar por esa experiencia. (Informante 1, 2014)

En el caso del personal subalterno, este proceso viene a través de la inducción por parte del personal más antiguo que administra los sistemas de abastecimiento, por lo general un suboficial superior. Es decir, el personal policial con muchos años en la organización se encarga de transmitir al personal nuevo la forma en que funciona el sistema de abastecimiento de combustible, aprovechando el carácter vertical de la organización policial expuesto anteriormente (Acha, 2004).

Entonces, es un superior con un subalterno, y basta que un superior de una indicación, y los subalternos, para quedar bien, lo aceptan. (Informante 9, 2015)

\section{Mecanismos de control en favor de la venta del combustible}

Si un efectivo policial va a ingresar en el sistema de administración de combustible, ello implica que necesariamente incurrirá en delitos que deberían ser sancionados, por lo tanto, una de sus principales preocupaciones será cómo manejar tal transgresión en los órganos de inspectoría y disciplina. Es decir, cómo va a reaccionar el Comando cuando se descubran las infracciones. En este caso, los nuevos policías aprenden que las faltas relacionadas con la venta del combustible no van a ser sancionadas, sino "corregidas». Ello significa que las supervisiones que realicen los órganos de inspección no se orientan a descubrir si se ha hecho o no un uso incorrecto del combustible para sancionar la infracción, sino a exigir que se cumpla con la formalidad de los registros.

Conociendo la realidad, es complicado que la Inspectoría sancione drásticamente este tipo de economías, por lo general dan advertencias para que se pongan al día los cuadernos, se reparen las unidades, etcétera. (Informante 8, 2015)

O sea, como que, si se va a verificar la administración, simplemente se llega a decir: «Bueno, ¿están cumpliendo con el abastecimiento? Sí. ¿Hasta cuándo han abastecido? Hasta ayer. Ya ¿Cuál es su vehículo? Este. ¿Está funcionando? Sí. Ya, ok», y ahí nomás quedó. (Informante 1, 2014)

Lo visto permite afirmar que al interior de la organización se produce una especie de "puesta en escena» en la cual los órganos disciplinarios solo se preocupan por verificar que se cumpla con los requerimientos formales, es decir, que los cuadernos y documentos de registro estén al día, aunque se sabe que lo más probable es que estos contengan información falsa. Esta política de control tan laxa tiene como objetivo primordial no entorpecer el cumplimiento de las funciones policiales para 
que, de esa manera, la evaluación sea sobre los resultados que cada dependencia policial obtenga: si una unidad tiene buenos récords de capturas de delincuentes, mantenimiento del orden público, etcétera, se continúa con la misma dotación del combustible, de suceder lo contrario, el recurso se recorta.

O sea [el general jefe de la Región), exigía que todos tengan resultados operativos. Entonces se dio cuenta de que no había ese rendimiento productivo [en un grupo de unidades policiales], entonces no justificaba que tengan, pues, tanta cantidad de combustible, cuando se daba cuenta de que sus recorridos no iban a ser mayores, ¿̨no? Y ya, pues, o sea, [efectuó] una buena racionalización [les cortó a la mitad la dotación de combustible]. (Informante 1, 2014)

Como se puede observar, estas normas ocultas sí procuran, al menos en principio, que se cumplan los objetivos organizacionales orientados a la ciudadanía. Pero a la vez, dejan la puerta abierta para cualquier otro uso arbitrario de los recursos, que será tolerado y permitido en la medida en que se justifiquen en la búsqueda del cumplimiento de los objetivos de la organización.

Por otro lado, un sistema cuya operatividad y viabilidad se sostiene en la transgresión de las normas escritas es un sistema expuesto a la delación. Considerando que este uso discrecional de la gasolina constituye un delito ${ }^{16}$, los miembros de la organización no pueden arriesgarse a que algunos de sus integrantes comiencen a denunciar estas transgresiones, así que, además del proceso de normalización interna, se debe procurar establecer una serie de premios y castigos para que nadie se salga del sistema. En otras palabras, la organización establece mecanismos para proteger a sus miembros de cualquier posibilidad de denuncia interna.

Si todas las unidades de la PNP pasan por los mismos problemas de provisión dispar de insumos y repuestos, aquel que no quiera participar del sistema se convierte en una amenaza. Al interior de la policía existen formas de represalia, no únicamente para los que delatan, sino incluso para los que se resisten a participar del sistema de administración irregular del combustible. Dentro de la organización existen historias sobre jefes de unidad que fueron sacados de sus cargos por no querer formar parte del sistema, es decir, por no utilizar el combustible para solucionar las carencias de la unidad. Estas historias, verdaderas o no, crean narrativas al interior de la institución que comunican la necesidad de la existencia del sistema, y de los efectos de no participar en él:

Hay intimidación: «si tú no arreglas el carro, te bajo del patrullero y te hago patrullar caminando. Si no arreglas el carro, te mando a la oficina». (Informante 13, 2016)

16 La mayoría de casos denunciados públicamente por el uso indebido de combustible acaban con penas de prisión efectiva. 
Sin embargo, aunque ser marginados del sistema de uso irregular del combustible limita las posibilidades de los efectivos policiales de avanzar en sus carreras, es al final una represalia benigna si se compara con lo que ocurre con los delatores. En el transcurso de la investigación se pudo conocer el caso de un suboficial que trabajaba en la Dirección contra la Corrupción de la PNP, que fue convocado en secreto por un fiscal, junto con otro efectivo subalterno, para una intervención a una unidad policial ${ }^{17}$. Ambos suboficiales se enteraron de que la intervención sería en una unidad policial solo minutos antes; a pesar de ello, cuando se les vio participando de la inspección junto con un fiscal, se les consideró como traidores al interior de la PNP y las consecuencias para ellos fueron absolutamente perjudiciales:

[...] Al haber participado nosotros en esta intervención como apoyo de la fiscalía, hemos sido objeto de amenazas inclusive por parte de los oficiales. (Informante $3,2014)$

Según lo que el entrevistado indica, lo que se esperaba era que, apenas se enteraron de que la intervención sería en una unidad policial, dieran la voz de alerta para prevenir a sus compañeros. Al no haber puesto en conocimiento de sus compañeros la inminencia de la intervención, fueron etiquetados como «malos policías» dentro de la organización y fueron excluidos del grupo y enviados a unidades no deseadas.

[...] Es como que quiere decir que primero le avisemos a dónde se va a actuar o accionar en contra de la policía, como quien puede advertir «avisemos primero al delincuente que le vamos a ir a intervenir para que no robe» [...]. (Informante 3, 2014)

Aquel efectivo policial que dice «señor, usted tiene que dejar sus tres galones de combustible para reparar el vehículo», el efectivo policial directamente firma, y el otro dice «no, yo no voy a firmar», «ah, no vas a firmar». Bien, lo sacan de ahí, la próxima rotación lo mandan a cualquier [lugar], lo sacan, lo van a excluir. Igualito va a suceder con el mismo jefe que no es parte, va a ser excluido y quitado. (Informante 2, 2014)

Por otro lado, para mantener la cooperación de todos los policías dentro del sistema, también se juega con los posibles beneficios para los que sí aceptan formar parte. Debido a que el número de vehículos policiales es limitado y que, por ende, no todos los efectivos pueden ser parte del abastecimiento, se genera una competencia entre los policías subalternos para poder participar en el sistema. Entre las

17 Debido al elemento del «pacto de silencio» propio de la cultura organizacional de la PNP, es particularmente difícil investigar este tipo de casos, pues las delaciones ocurren con muy poca frecuencia, y cuando ocurren, se mantienen en secreto. 
labores que corresponden a los suboficiales seleccionados está la de encargarse del manejo del combustible, por lo que una actitud favorable con el sistema establecido es una condición necesaria para ser elegidos. Una vez que ya se forma parte del sistema, y dependiendo de lo solícito o eficiente que resulte el efectivo en la tarea, hay altas probabilidades de gozar siempre de los beneficios ofrecidos ${ }^{18}$, en tanto es usual que los oficiales se hagan acompañar a lo largo de sus carreras por al menos un suboficial de su confianza.

Otra forma de mantener el sistema seguro es a través del control sobre los órganos de inspectoría. La inexistencia de especialidades en la policía garantiza un trato benevolente con los infractores, en tanto los que son inspectores en un momento determinado, en uno o dos años se convertían en encargados de una unidad operativa y, por lo tanto, susceptibles de ser fiscalizados. De esta manera, el hecho de que los supervisores de hoy vayan a convertirse en los supervisados de mañana, favorece la «solidaridad» y el "pacto de silencio» al interior de la organización policial.

Así es, yo puedo ser comisario por dos años y al siguiente me voy a trabajar a Inspectoría. (Informante 2, 2014)

El sistema y los mecanismos de control establecidos están diseñados para evitar que se investiguen este tipo de irregularidades, pero eso no implica que no existan expedientes de este tipo en los órganos de investigación y que no se sancione a policías por hechos de este tipo. Es decir, el sistema es susceptible de perturbaciones cuando las acusaciones vienen desde afuera de la PNP ${ }^{19}$. Ello explica el hecho de que sí se hayan conocido denuncias por robo de combustible en los últimos años por los medios de comunicación.

Y salvo... ¿cuándo lo han hecho? [que la Inspectoría de la PNP investigue casos de uso irregular de combustible]. Cuando recién han salido denuncias en medios de comunicación. Entonces dicen «el comisario tal, de tal lugar, está...», entonces ahí recién Inspectoría se aboca, porque ya hay una denuncia de por medio. (Informante 1, 2014)

\footnotetext{
18 Como los recursos generados por la venta del combustible, la capacidad de negociar permisos para sí mismos, o tener una menor carga de trabajo que los demás efectivos.

19 De acuerdo con algunos testimonios, también pueden venir desde dentro, cuando se producen enfrentamientos entre grupos al interior de la organización como los que provienen de las antiguas escuelas policiales: Guardia Civil, Policía de Investigaciones y Guardia Republicana, es decir, los llamados «código I», «código II» y «código III», respectivamente. Estos enfrentamientos ocurren sobre todo en procesos de ascenso o cuando el oficial que asume la Dirección General de la PNP busca privilegiar a sus compañeros de «código».
} 


\section{¿Corruptos o eficientes?}

Aunque la venta irregular del combustible se sostenga —al menos discursivamente- sobre las carencias organizacionales descritas, estas no explican las formas en las que dicha práctica se ha propagado al interior de la policía. La cultura organizacional de la PNP establece patrones de comportamiento que, en la búsqueda de cumplir con los deberes de la organización, terminan generando los mecanismos de refuerzo que aseguran la institucionalización de la venta ilícita del combustible. Así, por ejemplo, el "pacto de silencio" exige que ningún oficial denuncie a otro sin importar si participa o no directamente de la actividad. El caso de los policías castigados por «traidores» ejemplifica lo arraigada que está esta norma al interior de la PNP. Por otro lado, la verticalidad propia de instituciones jerárquicas como la PNP explica por qué los nuevos miembros terminan acatando las órdenes de sus superiores sin importar que estas sean ilegales.

Se ha constatado que la desigual distribución de insumos requeridos para la operatividad de los vehículos policiales es el punto sobre el cual se construyen los discursos que legitiman la venta ilegal de combustible para suplir tales carencias. Así, este acto de corrupción es aceptado porque permite cumplir con los objetivos institucionales de la PNP a través del patrullaje. Cuando sucede de esta manera, las definiciones clásicas que asocian a la corrupción con el beneficio privado y el deterioro del bien común (Nye, 1967; Tanzi, 1998; Soto, 2003) parecen no recoger de manera adecuada la realidad; por el contrario, una propuesta de definición como la de Huber, que considera que los actos de corrupción pueden aparecer para corregir fallos o carencias en las burocracias estales, adquiere mayor pertinencia y potencial explicativo para el caso analizado (Huber, 2008).

Mientras que la opinión pública sobre la policía se construye sobre las denuncias propaladas por los medios de comunicación, que exponen a los transgresores como «manzanas podridas» que buscan su beneficio personal enriqueciéndose con la venta del combustible, la legitimidad y autoridad del cargo de policía no se ve alterada para los miembros de la PNP, pues se justifican en el hecho de que no buscan un beneficio privado al vender el combustible, sino más bien obtener los recursos necesarios para asegurar el cumplimiento de sus funciones individuales e institucionales. En otras palabras, a pesar de que la sociedad peruana los percibe como una organización corrupta, en general los policías no ven mermada su autoridad por la venta ilegal del combustible ya que consideran que son eficientes al gestionarla de una manera tal que les permite cumplir con sus funciones.

Por todo lo mencionado, no es posible clasificar toda venta ilegal de combustible como casos de delitos directos dentro de la tipología de Roebuck y Barker (1974), es decir, no necesariamente estamos frente a un caso de venta de recursos públicos para obtener un beneficio personal, sino que, de acuerdo con los testimonios recogidos, 
podríamos estar frente a un caso de "corrupción por causa noble» o "corrupción blanca». Para clasificar la venta de combustible en una u otra categoría dentro de la tipología, resulta entonces necesario, tal como señala Leys (1965), conocer las motivaciones de cada policía transgresor.

Para responder a la pregunta "¿corruptos o eficientes?» resulta necesario hacer una precisión conceptual dentro de la categoría de corrupción blanca, por lo que planteamos los conceptos «corrupción por eficacia» y "corrupción por eficiencia». De un lado, la «corrupción por eficacia» referiría a actos de corrupción orientados principalmente al cumplimiento de las metas de la institución al margen del costo económico o social que ello pudiera implicar. Por ejemplo, alterar evidencia con el fin de aumentar el número de detenidos ${ }^{20}$. En cambio, la "corrupción por eficiencia» sería más sofisticada, en la medida en que se referiría a los actos que involucran un uso indebido del poder o de la autoridad, igualmente orientados al cumplimiento de las metas organizaciones, pero a través de un uso racional de los recursos. De esta manera, la venta irregular de combustible analizada, aparece como un caso de "corrupción por eficiencia», donde la consecución de los objetivos organizacionales pasa por una mejora en la operatividad de la institución mediante una redistribución de los recursos. Tal como vimos en el caso de la venta ilegal de combustible en la PNP, detrás de dichos actos subsiste una racionalidad orientada principalmente a optimizar el funcionamiento operativo de la organización y que, al mismo tiempo, permite a sus miembros tejer narrativas que justifican los actos de corrupción.

Finalmente, podemos suponer que el concepto de corrupción por eficiencia no es un fenómeno exclusivo de la PNP, sino que también puede tener lugar en otras organizaciones del Estado. El estudio de Cerna (2017), que retoma lo planteado por Lipsky (2010), explica cómo la burocracia de la calle, aquella con la que los ciudadanos interactúan cotidianamente, se encuentra mediada entre las exigencias del Estado, las demandas de la población y los distintos tipos de limitaciones de recursos, información, conocimientos, etcétera, dando lugar a la creación de sus propios mecanismos, reglas y rutinas informales para sortear dichas limitaciones, bajo la premisa de que «se hace lo mejor que se puede». Es posible, entonces, entender estos casos no como una transgresión en contra de los objetivos del sistema, sino como fenómenos que ocurren como consecuencia de este sistema. De aquí la importancia de preguntarnos por las funciones que cumple la corrupción al institucionalizarse dentro de una organización y, principalmente, sobre cuál es el sentido que cobra para los agentes que reproducen estas prácticas.

\footnotetext{
20 Maurice Punch (1985) desarrolla la categoría de «sembrado», que podría considerarse como un caso de "corrupción por eficacia». El sembrado consiste en plantar evidencia, por ejemplo, «sembrando" droga en casos de investigación antinarcóticos o colocando un arma en la escena de un tiroteo, de manera que se justifique el que un efectivo policial abata a un delincuente.
} 


\section{Conclusiones}

A pesar de que siempre se ha considerado difícil abarcar las dimensiones de la corrupción, gracias a la sofisticación de los métodos de control del abastecimiento de combustible se ha podido construir una herramienta para aproximarnos a la dimensión de esta práctica corrupta dentro de la PNP de Lima Metropolitana. Dados los reportes de rendimiento del combustible supuestamente abastecido para una muestra de 5031 vehículos policiales entre setiembre de 2014 y diciembre de 2015, podemos saber que el 59\% de las unidades arrojó reportes altamente sospechosos, es decir, reportes que no son consistentes con la realidad y que, por lo tanto, delatan una simulación del proceso, es decir, la gasolina no fue abastecida realmente en los vehículos policiales, y por lo tanto, da cuenta de la comisión de un delito. Con ello, se puede inferir que las prácticas de corrupción ligadas a la dotación de combustible no son casos aislados de «manzanas podridas» al interior de la PNP, sino que se trata de un comportamiento generalizado.

El uso ilegal que se da al combustible dentro de la PNP representa un ejemplo de corrupción policial donde la práctica corrupta —en este caso la venta ilícita del combustible- no se realiza necesariamente en contra de los intereses y funciones de la organización policial, ni tampoco de los de la sociedad en general. Por el contrario, las carencias organizacionales de otros insumos necesarios para los vehículos conducen primero a la transgresión de las normas y luego a la normalización de dicha transgresión, al propiciar discursos que legitiman la venta del combustible para suplir dichas carencias y así cumplir con la labor policial del patrullaje, enfocada en asegurar la seguridad ciudadana.

El origen de las narrativas que justifican y legitiman la sustracción y venta de combustible está en la deficiente distribución de recursos al interior de la PNP. Que la provisión de combustible no guarde relación con la provisión de repuestos y suministros deja abierta la posibilidad al uso irregular del combustible, de manera que no solo existe la necesidad de implementar políticas reactivas a las prácticas corruptas, sino también políticas que neutralicen los discursos que justifican dichas prácticas al interior de la PNP. Una vez anuladas las narrativas de legitimización, se podría esperar mejores resultados de las políticas de control.

Si bien no queda claro que todos los casos de sustracción y venta del combustible de unidades policiales busquen exclusivamente el beneficio de la organización, sí es más evidente que la práctica se ha normalizado y aceptado bajo ese discurso. De esa manera, los casos de transgresión a las normas de combustible pueden caer en cualquier punto entre buscar el cumplimiento de las metas organizacionales y buscar el enriquecimiento individual. Así, no hay una única categoría prevista dentro de la tipología de corrupción donde este caso de corrupción calce sin dejar lugar a dudas, pues bien podría tratarse de casos de corrupción por causas 
nobles ${ }^{21}$ como de actividades criminales directas. Las particularidades del tipo de corrupción estudiado nos permiten sugerir la introducción de la categoría de "corrupción por eficiencia» como una herramienta analítica para entender estas dimensiones del fenómeno de la corrupción.

También se ha demostrado cómo al interior de las organizaciones las normas informales fundadas en las prácticas cotidianas pueden llegar a tener un peso mayor que las normas formales, pues se generan sobre la base de la retroalimentación entre los actos y los discursos, los mandatos y la capacidad de ejecución, así como la conciliación entre las demandas de la sociedad y las demandas del grupo al que se pertenece. En última instancia, si bien no se justifica de manera absoluta ni para todos los casos, el proceso de normalización de la transgresión explica cómo es que un policía puede infringir la ley y, aun así, ser y sentirse capaz de vigilar que el resto de la sociedad no se desvíe de las normas establecidas formalmente, puesto que es posible que, cuando transgredió las normas, dentro de su propia narrativa no estuviese buscando un beneficio personal, sino más bien cumplir con los objetivos de la organización. No obstante, aun cuando el tipo de corrupción analizado procura un beneficio público, la normalización de la práctica aumenta la tolerancia a la corrupción al interior de la organización y, por lo tanto, no solo no soluciona las fallas del sistema sino que las institucionaliza, y deja la puerta abierta para otras formas de transgresión orientadas, esta vez sí, al beneficio personal.

\section{REFERENCIAS BIBLIOGRÁFICAS}

Acha, E. (2004). Cultura organizacional: vigencia e importancia de un concepto para la reforma de la policía. Debates en Sociología, 140-159.

América TV (2014a). Presentan sistema electrónico que controla abastecimiento de gasolina en la PNP. Recuperado de http://www.americatv.com.pe/noticias/actualidad/presentan-sistema-electronico-que-controla-abastecimiento-gasolina-policia-n 145440

América TV (2014b). Cusco: detuvieron a dos policías acusados de robar combustible. Recuperado de http://www.americatv.com.pe/noticias/actualidad/ cusco-detuvieron-2-policias-acusados-robar-combustible-n132504.

Ashforth, Blake E. y Anand, V. (2003). The normalization of corruption in organizations. Research in Organizational Behavior, 1-52. https://doi.org/10.1016/ S0191-3085(03)25001-2

Boehm, F. y Lambsdorff, J. (2009). Corrupción y anticorrupción: una perspectiva neo-institucional. Revista de Economía Institucional, 45-72.

21 Para Sayed y Bruce (1998), la corrupción blanca se refiere exclusivamente al uso de medios ilegítimos para fines legítimos. 
Cerna, D. (2017). Acá no hay ventanillas: la burocracia de la calle en los programas sociales. Lima: Instituto de Estudios Peruanos.

Chan, J. (1996). Changing police culture. The British Journal of Criminology, 109-134. https://doi.org/10.1093/oxfordjournals.bjc.a014061

Dacoba, E. (2009). Los efectos económicos de la corrupción. Tesis de pregrado. Pontificia Universidad Católica Argentina.

El Comercio (2011). Cajamarca: policías habrían robado combustible de sus propias patrullas. El Comercio. Recuperado de http://elcomercio.pe/peru/lima/cajamarcapolicias-habrian-robado-combustible-sus-propias-patrullas-noticia-843105.

El Comercio (2015). Robo de Gasolina en la Policía: Nueva modalidad al descubierto. Recuperado de https:/elcomercio.pe/lima/ robo-gasolina-policia-nueva-modalidad-descubierto-359038

Helmke, G. y Levitsky, S. (2003). Informal institutions and comparative politics: A research agenda. Working paper. Kellogg Institute.

Huber, L. (2008). Romper la mano. Una interpretación cultural de la corrupción. Lima: Instituto de Estudios Peruanos, Proética.

Kleining, J. (2002). Rethinking noble cause corruption. International Journal of Police Science \& Management, 4(4), 287-314. https://doi.org/10.1350/ijps.4.4.287.10877

La República (1 de julio de 2017). Detienen a comisario y dos policías por robar gasolina. Recuperado de http://larepublica.pe/ politica/891357-detienen-comisario-y-dos-policias-por-robar-gasolina

Leys, C. (1965). What is the Problem About Corruption? The Journal of Modern African Studies, 215-230. https://doi.org/10.1017/S0022278X00023636

Lipsky, M. (2010). Toward a Theory for Street-level Bureaucracy. Congreso Anual de la Asociación Americana de Ciencia Política (APSA), 2-6.

May, C. (2009). Implementing, Embedding and Integrating Practices: An Outline of Normalization Process Theory. Sociology, 43(3), 535-554. https://doi. org/10.1177/0038038509103208

Ministerio del Interior (2014). Policía Nacional tiene un nuevo sistema de control de abastecimiento de combustible. Lima. Recuperado de https:/www.mininter.gob.pe/ content/polic\%C3\%AD-nacional-tiene-un-nuevo-sistema-de-control-de-abastecimiento-de-combustible.

Nye, J. (1967). Corruption and political development. American Political Science Review, 417-427. https://doi.org/10.2307/1953254

Proética (2017). Décima Encuesta Nacional sobre percepciones de la corrupción en el Perú. Lima: Ipsos.

Punch, M. (1985). Conduct Unbecoming: The Social Construction of Police Deviance and Control». The Canadian Journal of Sociology, 11(3), 322-324. https://doi. org/10.2307/3341111

Quiroz, A. W. (2013). Historia de la corrupción en el Perú. Lima: IEP.

Reiner, R. (2010). The Politics of the Police. Oxford University Press. https://doi.org/10.1093/ he/9780199283392.001.0001 
Roebuck, J. (1974). A Typology of Police Corruption. Social Problems. https://doi. org/10.2307/799909

Salés, P. P. (1 de setiembre de 2014). Policías se dedican al hurto sistemático de combustible. La República. Recuperado de http://www.larepublica.pe/02-09-2014/ policias-se-dedican-al-hurto-sistematico-de-combustible

Sayed, T. Y. y Bruce, D. (1998). Inside and Outside the Boundaries of Police Corruption. African Security Review, 7(2). https://doi.org/10.1080/10246029.1998.9627846

Soto, R. (2003). La corrupción desde una perspectiva económica. Estudios Públicos, 23-62.

Tanzi, V. (1998). Corruption around the World: Causes, Consequences, Scope, and Cures. Working Paper of the International Monetary Fund. International Monetary Fund. https://doi.org/10.2307/3867585

Toca, C. y Carrillo, J. (2009). Asuntos teóricos y metodológicos de la cultura organizacional. Civilizar, 117-136. https://doi.org/10.22518/16578953.711

Trevińo, L. K. y Youngblood, S. (1990). Bad apples in bad barrels. A causal analysis of ethical decition-making behavior. Journal of Applied Psychology, 378-385. https://doi. org/10.1037/0021-9010.75.4.378

Tudela, P. (2010). Cultura policial y control interno. Revista Estudios Policiales, 74-107.

UNOD (2005). Handbook on Practical Anti-Corruption Measures for Prosecutors and Investigators. UNDC. Recuperado de https://www.unodc.org/pdf/corruption/ publications_handbook_prosecutors.pdf 